\title{
Mutations in Escherichia coli that Relieve Catabolite Repression of Tryptophanase Synthesis. Tryptophanase Promoter-like Mutations
}

\author{
By D. F. WARD AND M. D. YUDKIN \\ Microbiology Unit, Department of Biochemistry, University of Oxford, \\ Oxford $O X_{\mathrm{I}} 3 Q U$
}

(Received 8 May 1975; revised I I June 1975)

\section{SUMMARY}

From a strain lacking adenyl cyclase and the catabolite-sensitive gene activator protein, two mutants were isolated that can synthesize tryptophanase. Each mutation is extremely closely linked to the tryptophanase structural gene. The mutations differ from one another in the rate of synthesis of tryptophanase that they permit in the genetic background in which they were isolated; they differ from one another and also from the wild type in the maximum rate of synthesis of tryptophanase that they permit in a genetic background with intact adenyl cyclase and catabolitesensitive gene activator protein. Both mutations appear to lie in the tryptophanase promoter.

\section{INTRODUCTION}

The accompanying paper (Yudkin, 1976) shows that the synthesis of tryptophanase in wild-type Escherichia coli is absolutely dependent on an intact cyclic AMP-catabolitesensitive gene activator (c-AMP-CGA) protein system. Nevertheless, from mutants that lack both adenyl cyclase and the CGA protein it is possible to isolate revertants that are able to synthesize tryptophanase. We describe two such revertants and present evidence that suggests that the mutations responsible for the revertant phenotype are in the promoter for the tryptophanase structural gene.

\section{METHODS}

Genetic symbols. These are as in Taylor \& Trotter (1972), and include crp (the structural gene for the catabolite-sensitive gene activator protein), cya (the structural gene for adenyl cyclase), and tha (the structural gene for tryptophanase). Ind ${ }^{+}$strains are capable of using indole as a source of tryptophan in the absence of 5 -methyltryptophan; Val ${ }^{\mathbb{R}}$ strains are valine-resistant.

Genetic methods, and differential rate of tryptophanase synthesis. These were as described by Yudkin (1976).

Bacteria. Strain MY57I ( $\left.\mathrm{F}^{-} t r p^{\text {del }} A C 9\right)$ was from Dr C. Yanofsky, strain MY6I8 (F- $\mathrm{F}^{-}$crp868 metB strA) from Dr J. Scaife, and strain MY613 ( $\mathrm{F}^{-}$thr leu his arg trp Ind ${ }^{+}$cysG lac Y gal $x y l$ mal man strA) from Dr M. Jones-Mortimer. Strain MY28I $\left(\mathrm{F}^{-} \operatorname{trp}^{\mathrm{del}} A C 9\right.$ tna $\left.\mathrm{Val}^{\mathrm{R}}\right)$ was constructed from strain MY57I by the method described by Yudkin (1976).

Strain MY283 (F-thr his arg trp ${ }^{\text {del }} A E$-tonB ${ }^{\text {del }}$ lac $Y$ gal $x y l$ mal man strA $\mathrm{Val}^{\mathrm{R}}$ cya855 crp868) was constructed in the following way. Spontaneous ton $B$ deletions of strain MY613 were selected (Gottesman \& Beckwith, 1969), and an Ind- derivative was purified; this was shown to carry a deletion of all the trp structural genes (Jackson \& Yanofsky, 1972). This strain was transduced to $l e u^{+}$and then to $c y a 855 \mathrm{Val}^{\mathrm{B}}$ (Yudkin, 1976); the resulting strain is 
MY279. To introduce $c r p^{-}$we made use of three facts (our unpublished work): (i) neither cya- nor $c r p^{-}$strains are able to synthesize tryptophanase in L-broth; (ii) $c y a^{-}$strains synthesize tryptophanase and give a positive test for indole when grown overnight in L-broth supplemented with I mM-c-AMP, whereas $c r p^{-}$strains do not; (iii) $c r p$ can be readily cotransduced with cysG. We therefore transduced strain MY279 with phage PI grown on strain MY6I8, selected $c y s G^{+}$recombinants, and retained one that was unable to make tryptophanase in the presence of c-AMP; this is strain MY283. We were able to recover both $c y a^{-}$and $c r p^{-}$from a transducing lysate of phage PI grown on the strain.

Media. These were as described previously (Yudkin, 1976). L-Threonine, L-histidine and L-arginine ( $50 \mu \mathrm{g}$ of each $/ \mathrm{ml}$ ) were added to minimal media for all strains that required these amino acids. 'Minimal medium' is to be taken as including these amino acids when they are required.

\section{RESULTS AND DISCUSSION}

\section{Isolation and properties of the revertants}

Strain MY283 carries a deletion of all the trp structural genes. To grow on minimal medium containing indole and 5-methyltryptophan it must therefore make tryptophanase (Yudkin, 1976). However, as it carries mutations in the structural genes for adenyl cyclase and the CGA protein, it will be able to make tryptophanase only by acquiring a means of expressing tha that does not depend on the c-AMP-CGA system.

Cells of strain MY283 were harvested from stationary culture in L-broth, washed twice in $0.9 \% \mathrm{NaCl}$ and plated on glucose-minimal medium with indole and 5-methyltryptophan. After two days at $37^{\circ} \mathrm{C}$, colonies appeared at about $\mathrm{I}$ to $2 / 10^{10}$ cells plated. Two strains, each arising from a separate broth culture of strain MY283, were purified and named MY627 and MY634. They are similar in the following respects: they make colonies in 1 to 2 days at $37^{\circ} \mathrm{C}$ on glucose-minimal medium with indole and 5-methyltryptophan, or on the same medium supplemented with gluconate; they fail to grow on glycerol-tryptophanminimal or on arabinose-tryptophan-minimal medium; they make $\beta$-galactosidase, when induced, at the same very low rate as the strain MY283 from which they are derived. They differ in their rate of synthesis of tryptophanase: in glucose-tryptophan-minimal medium strain MY627 makes I5 units/mg protein and strain MY634 makes 4I units. (Strain MY283 makes less than I unit/mg protein.)

\section{Genetic characterization}

The above results suggest that the mutations in strains MY627 and MY634 permit the expression of tha notwithstanding the $c y a^{-}$and $c r p^{-}$mutations, but do not allow other catabolite-sensitive systems to be expressed. Since the c-AMP-CGA protein system is thought to work by interaction with the promoter, and since it is known that some lac promoter mutations permit lac to be expressed in a cya $^{-}$or crp $^{-}$background (Arditti, Grodzicker \& Beckwith, 1973), an obvious possibility is that strains MY627 and MY634 contain tryptophanase promoter mutations. To test this idea, we did a number of transductions designed to show whether the mutations are located near tna.

Phage PI grown on strain MY634 was used to transduce strain MY57I to grow on glucoseminimal medium supplemented with indole and 5-methyltryptophan and with gluconate. [Strain MY57I cannot normally grow on this medium because it carries a $\operatorname{trp}$ deletion and because the severe catabolite repression imposed by the glucose and gluconate prevents the synthesis of sufficient tryptophanase to convert indole to tryptophan (Yudkin, 1976); since strain MY634 is able to grow in these circumstances we presumed that it would be able 
to donate this ability to strain MY571.] By replicating the transductants to medium supplemented with valine we showed that $26 \%$ had become valine-resistant, and by replicating to glycerol-tryptophan-minimal medium we showed that $7 \%$ had inherited cya- from the donor.

In a similar way we showed that the mutation that permitted tryptophanase synthesis in strain MY627 was $19 \%$ co-transducible with valine resistance and $4 \%$ co-transducible with cya.

These results show that the two mutations are close to the ilv locus in which valine resistance maps, on the opposite side of $i l v$ from cya, and they suggest that the mutations may be very near to tna (see Fig. I of Yudkin, 1976).

To determine the linkage to tna, we used the phage preparation grown on strain MY634 to transduce strain MY28I (trp ${ }^{\text {del }}$ tna ${ }^{-}$) to Tna ${ }^{+}$. Recombinants were selected on indole-5methyltryptophan-minimal medium with glycerol as carbon source (on which $t r p^{\text {del }}$ strains can grow provided that they are $\mathrm{Tna}^{+}$); they were then streaked on to the same medium and on to indole-5-methyltryptophan medium with glucose and gluconate, to determine whether they had also inherited the mutation that permits expression of tna in the presence of these carbon sources. Of 117 transductants tested, all were able to grow on the latter medium. (When we repeated this experiment using phage $P_{1}$ that had been grown on another revertant strain that is indistinguishable from strain MY634 we found that 399 out of 401 transductants tested were able to grow on glucose-gluconate-minimal medium with indole and 5-methyltryptophan.) Similarly, 102 out of $\mathrm{IO}^{2} \mathrm{Tna}^{+}$recombinants obtained by transducing strain MY28I with phage Pr grown on strain MY627 were able to express tna in the presence of glucose plus gluconate.

At first sight these results suggest that the mutations that permit strains MY627 and MY634 to express tna are more than $99 \%$ co-transducible with the tna structural gene. However, since the mutations in strains MY627 and MY634 are co-transducible to a slight extent with $\mathrm{cya}^{-}$, selection of $\mathrm{Tna}^{+}$transductants on medium containing glycerol as sole source of carbon will have excluded a small fraction of potential transductants (those few that have inherited cya as well as tna $a^{+}$from the donor). In spite of this slight bias in the selection, we think it safe to conclude that the mutations in strains MY627 and MY634 are extremely closely linked with tna. This conclusion is compatible with their being in the tryptophanase promoter.

An alternative possibility is that in each mutant the tna gene has been duplicated and one copy has become translocated and attached to a promoter that is independent of the c-AMP-CGA protein system. This suggestion is incompatible with the following facts: (i) By plating out the mutant strains on nutrient agar and replicating several thousand colonies to glucose medium with indole and 5-methyltryptophan we showed that our mutants were stable, whereas duplication-translocation events in $E$. coli are characterized by instability (Hill et al. 1969; Jackson \& Yanofsky, 1973); (ii) the co-transducibility between our mutations and valine resistance is the same as that between tna and valine resistance in the wild type (contrast the much decreased linkage between cysB and the translocated trp genes described by Jackson \& Yanofsky, 1973); (iii) the mutations are extremely closely linked to tna: if there were two copies of the tna gene in the mutants, transduction would frequently separate $t n a^{+}$from the mutations that lead to catabolite insensitivity. We think our results are best explained by assuming that the promoter of the tha gene has mutated in strains MY627 and MY634. We designate these mutated promoters tnaPI and tnaP2 respectively. 
Table I. Differential rates of tryptophanase synthesis in cya $a^{-}$crp $^{-}$and cya $a^{+}$crp $^{+}$backgrounds

\begin{tabular}{|c|c|c|c|}
\hline \multirow[b]{2}{*}{ Strain } & \multirow[b]{2}{*}{ Genotype } & \multicolumn{2}{|c|}{$\begin{array}{c}\text { Differential rate of trypto- } \\
\text { phanase synthesis (enzyme } \\
\text { units/mg protein) }\end{array}$} \\
\hline & & $\begin{array}{l}\text { Glycerol- } \\
\text { minimal } \\
\text { medium }\end{array}$ & $\begin{array}{l}\text { Glucose- } \\
\text { minimal } \\
\text { medium }\end{array}$ \\
\hline MY57I & $\operatorname{trp}{ }^{\mathrm{del}}$ tnaP ${ }^{+}$cya $a^{+}$crp ${ }^{+}$ & 173 & 13 \\
\hline MY627 & $\operatorname{trp}^{\mathrm{del}}$ tnaPI cya- crp & -* & 15 \\
\hline MY281/627 & $\operatorname{trp} p^{\mathrm{del}} \operatorname{thaP} \mathrm{I} c y a^{+} c r p^{+}$ & 57 & 31 \\
\hline MY634 & $\operatorname{trp} p^{\mathrm{del}} \operatorname{tnaP} 2$ cya $c r p^{-}$ & $-^{*}$ & 41 \\
\hline MY281/634 & $\operatorname{trp} p^{\text {del }} \operatorname{tnaP2} c y a^{+} c p^{+}$ & 105 & 42 \\
\hline
\end{tabular}

* Because of the absence of the c-AMP-CGA protein system, strains MY627 and MY634 do not grow in glycerol-minimal medium.

\section{Expression of the mutations in a cya ${ }^{+}$crp $^{+}$background}

We purified one recombinant from each of the transductions in which strains MY627 and MY634 were the donors and strain MY28I was the recipient. By measuring differential rates of tryptophanase synthesis in these recombinants, we were able to study the expression of tnaPr and tnaPz in a strain that had an intact c-AMP-CGA protein system. The results are presented in Table I, together with results of similar experiments in strain MY57I - the isogenic strain with a wild-type tna region.

thaPr, when placed in a $c y a^{+} c r p^{+}$background, is evidently far less susceptible to catabolite repression than a wild-type promoter; moreover, it promotes expression of tha in glycerol-minimal medium at only about one-third of the wild-type rate. tnaP2, when placed in a $c y a^{+} c r p^{+}$background, retains more sensitivity to catabolite repression, but in glycerolminimal medium it still promotes expression at only $60 \%$ of the wild-type rate. Thus both promoter mutations change the rate of expression of $t$ na whether they are in a cya crp $^{-}$ or in a $c y a^{+}$crp ${ }^{+}$background.

Neither of our two mutants corresponds closely to any of the large number of lac promoter mutants that have been described. lac promoter mutations fall into three general classes: (a) mutations that permit greatly diminished expression of lac in a $c y a^{+}$crp $^{+}$background but the same rate of expression as $\boldsymbol{P}^{+}$in a $c y a^{-}$crp- background (Beckwith, Grodzicker \& Arditti, 1972); (b) mutations that permit the wild-type rate of expression in a $c y a^{+} c^{+} p^{+}$ background but a much higher rate of expression than $P^{+}$in a $c y a^{-}$crp ${ }^{-}$background (Arditti et al. 1973); (c) mutations that allow a lower rate of expression than $P^{+}$in either a $c y a^{+} c r p^{+}$ or a cya- crp- background (Hopkins, 1974). Our mutants most nearly resemble lac mutants of class $(b)$, which were isolated by a procedure analogous to ours; but (unlike those) our mutations clearly affect the maximum level of gene expression in a cya ${ }^{+}$crp $^{+}$background. Beckwith et al. (1972) suggested that the lac promoter can be divided into two sites, of which one interacts with the CGA protein and is altered in mutants of class $(a)$, and the other interacts with the RNA polymerase and is altered in mutants of classes $(b)$ and $(c)$. Our results do not provide evidence for a clear demarcation between two such sites for the tryptophanase promoter, since the mutations abolish dependence on the c-AMP-CGA system and also diminish the maximum level of gene expression. Our mutations could be deletions covering both putative sites, but deletions of that sort would probably have a more drastic effect on the maximum level of tna expression. Again, our strains MY627 and 
MY634 may each carry two mutations; they were, however, isolated without the use of a mutagen. In any case the lac promoter is perhaps not very similar to the tryptophanase promoter, since lac is expressed at $2 \%$ of the wild-type rate even in cya ${ }^{-}$cr $^{-}$strains (Beckwith et al. 1972), whereas the corresponding figure for tha is less than $0.1 \%$.

We thank Dr M. Jones-Mortimer, Dr J. Scaife and Dr C. Yanofsky for gifts of strains.

\section{REFERENCES}

ArditTI, R., Grodzicker, T. \& BeCKwITH, J. (1973). Cyclic adenosine monophosphate-independent mutants of the lactose operon of Escherichia coli. Journal of Bacteriology II4, 652-655.

BeCKWTTH, J., Grodzicker, T. \& ARDITTI, R. (1972). Evidence for two sites in the lac promoter region. Journal of Molecular Biology 69, 155-160.

GotTESMAN, S. \& BeCKWTTH, J. R. (1969). Directed transposition of the arabinose operon: a technique for the isolation of specialized transducing bacteriophages for any Escherichia coli gene. Journal of Molecular Biology 44, I1 7-127.

HILL, C. W., FourdS, J., Soll, L. \& BERG, P. (I969). Instability of a missense suppressor resulting from a duplication of genetic material. Journal of Molecular Biology 39, 563-581.

HopKINS, J. D. (1974). A new class of promoter mutations in the lactose operon of Escherichia coli. Journal of Molecular Biology 87, 715-724.

JACKSON, E. N. \& YANOFSKY, C. (1972). Internal deletions in the tryptophan operon of Escherichia coli. Journal of Molecular Biology 71, 149-161.

JACKSON, E. N. \& YANOFSKY, C. (1973). Duplication-translocations of tryptophan operon genes in Escherichia coli. Journal of Bacteriology 116, 33-40.

TAYLOR, A. L. \& TrOTTER, C. D. (1972). Linkage map of Escherichia coli strain K12. Bacteriological Reviews $36,504-524$.

YUDKIN, M. D. (1970). Mutations in Escherichia coli that relieve catabolite repression of tryptophanase synthesis. Mutations distant from the tryptophanase gene. Journal of General Microbiology 92, 125-132. 